\title{
Análisis plástico y Ensayos de Losas multidireccionales de HRFA
}

\section{Plastic Analysis and Testing of multidirectional SFRC flag slabs}

\author{
A. Maturana $^{(*)}$, J. Canales $^{(*)}$, A. Orbe ${ }^{(*)}$, J. Cuadrado $^{(*)}$
}

\section{RESUMEN}

En este trabajo se realiza un estudio teórico- experimental de losas multidireccionales de hormigón reforzado con fibras de acero (HRFA). El estudio se enmarca en una aplicación de un edificio real usando HRFA en los forjados. Para la evaluación de la capacidad resistente de las losas, se realizan cálculos plásticos, tanto a nivel de sección como de estructura. A nivel de sección se emplea el diagrama tensión-deformación plástico perfecto, tomando como referencia los valores de las características resistentes del HRFA aportados en trabajos anteriores que emplearon fibras y dosificaciones similares. A nivel de estructura se emplea el método plástico de las líneas de rotura, que relaciona el momento último de la sección la carga de colapso plástico. La campaña experimental, consiste en ensayar seis prototipos en forma de loseta circular de $2 \mathrm{~m}$ de diámetro, verificando el valor de la resistencia de referencia empleada en los cálculos.

Palabras clave: Hormigón reforzado con fibras de acero; losas; análisis plástico; líneas de rotura; ensayos.

\section{ABSTRACT}

This paper presents a theoretical and experimental study of multidirectional steel fibers reinforced concrete slabs (SFRC). The study is based on a real building application using SFRC flag slabs. For the evaluation of the slabs bearing capacity, plastic calculations are performed both at section and structure levels. The section analysis uses the perfect plastic stressstrain diagram, with reference to the values of the strength characteristics of SFRC based on previous jobs that used similar fibers and dosages. In the structure analysis the plastic yield lines method has been used. This method relates the section last bearing moment and the plastic collapse load. The experimental campaign has consisted of the testing of six 2 $m$. diameter circular shaped slabs prototypes, and has allowed to verify the reference resistance used in the calculations.

Keywords: Steel fibers reinforced concrete; flag slab; plastic analysis; yield lines; testing.

(*) UPV-EHU Universidad del País Vasco

Persona de contacto/Corresponging author: aitor.maturana@ehu.es (A. Maturana)

Cómo citar este artículo/Citation: Maturana, A., Canales, J., Orbe, A., Cuadrado, J. (2014). Análisis plástico y Ensayos de Losas multidireccionales de HRFA. Informes de la Construcción, 66(535): e031, doi: http://dx.doi.org/10.3989/ic.13.021.

Licencia/License: Salvo indicación contraria, todos los contenidos de la edición electrónica de Informes de la Construcción se distribuyen bajo una licencia de uso y distribución Creative Commons Reconocimiento no Comercial 3.o. España (cc-by-nc). 


\section{INTRODUCCIÓN}

La utilización del hormigón reforzado con fibras persigue la mejora de los procesos constructivos, reduciendo la mano de obra de manipulación de armaduras in situ y optimizando los plazos de ejecución. Desde el punto de vista resistente, el material presenta isotropía, y aumenta la durabilidad ya que el armado volumétrico reduce las fisuras por retracción y por lo tanto el riesgo de corrosión (1).

El objetivo estructural principal de las fibras metálicas es dotar de capacidad resistente al hormigón después de su fisuración. Para ello las fibras actúan como un armado volumétrico que cosen y coartan la propagación de las fisuras en la matriz de hormigón. Por otro lado las fisuras aumentan en gran medida la ductilidad del material resultante, dotándole de mayor capacidad de deformación antes del colapso. Las fibras comienzan a trabajar tras el inicio de la fisuración, dotándole al material de una resistencia residual a tracción que influye significativamente en la capacidad a flexión del material. La adición de las fibras metálicas supone un aumento significativo de la tenacidad como mostraron Barros y Figuieras (2).

En la actualidad el uso del HRFA se ha extendido ampliamente en elementos que no poseen una finalidad puramente estructural (3). Las aplicaciones y experiencias existentes en el uso del HRFA con función estructural en forjados son escasas. El primer forjado dentro del ámbito europeo, se construyó íntegramente con HRFA en Bissen (Luxemburgo) y data del año 2004. Los estudios previos a su construcción los realizaron Massicotte y Moffat (4). Las pruebas y conclusiones asociadas al prototipo ensayado se recogen en (5) (6) y (7). En el ámbito español sólo existe una experiencia reseñable en este sentido, aunque consistió en una rehabilitación de un edificio industrial (8), dónde se ejecutó una losa de HRFA quedando embebidas unas viguetas metálicas existentes como nervios estructurales, por lo que no constituye un caso comparable al edificio que se describe en el presente trabajo.

Existen diversos métodos y ensayos para caracterizar la resistencia del HRFA. Los más empleados son los ensayos de flexotracción de probetas prismáticas, aunque en muchas ocasiones presentan elevadas dispersiones. El ensayo Barcelona de doble punzonamiento de Aguado et ál (9) y (10), reduce la dispersión, pero conlleva la dificultad de correlacionar las mediciones del ensayo con las resistencias a evaluar. Los ensayos de paneles realizados por Bernard (11) respecto a los de probetas prismáticas, presentan una menor dispersión de resultados, debido a que la superficie de rotura es mayor. El inconveniente principal radica en la elaboración de los paneles, y que han sido diseñados específicamente para su aplicación al hormigón proyectado.

No existe aún un modelo constitutivo relativo al comportamiento a tracción del HRFA que se imponga sobre los demás. Algunos estudios experimentales realizados por Blanco et ál (12) demuestran que los modelos recogidos en el anejo de fibras de la Instrucción del Hormigón estructural (EHE) (13) presentan una buena predicción del comportamiento del material de forma general.

El incremento de tenacidad que proporcionan las fibras al hormigón aumenta la aplicabilidad de los métodos de cálculo plástico. Esto es aún más evidente en el caso de placas o losas macizas de hormigón, mediante el método de las líneas de rotura (14).
En este trabajo se realiza un análisis plástico de las losas de forjado de un edificio real construido en HRFA. Para llevar a cabo este análisis, se describe y analiza una experiencia singular y pionera en España, consistente en la construcción del primer edificio de ámbito nacional dónde se ha aplicado el HRFA en losas de forjado apoyadas directamente sobre pilares, sustituyendo la armadura convencional por una dosificación de fibras metálicas.

Las losas, que se encuentran apoyadas directamente sobre los pilares, se analizan en estado límite último a nivel de sección y de estructura. A nivel de sección, empleando el diagrama tensión- deformación plástico perfecto propuesto por EHE, se obtiene el momento plástico último que es capaz de desarrollar la sección, aunque ello requiere conocer previamente la resistencia residual a tracción del HRFA. El valor de esta resistencia se ha tomado de las experiencias con fibras y dosificaciones similares realizadas por otros autores, y su valor se verificará mediante una campaña experimental posterior. A nivel de estructura, mediante el estudio de los diferentes mecanismos de colapso por el método de las líneas de rotura, se relaciona la carga de colapso superficial con el momento último de la sección, y se comprobará que su valor es menor que la carga de diseño actuante.

El estudio se completa con una campaña experimental que consiste en la rotura de seis prototipos en forma de loseta circular, de $2 \mathrm{~m}$ de diámetro y $200 \mathrm{~mm}$ de espesor. Los prototipos se han elaborando simultáneamente a la ejecución de la estructura del edificio analizado, con el mismo HRFA con el que se han ejecutado las losas, y necesariamente con los mismos procedimientos de ejecución, curado y endurecido a la intemperie. Los resultados de los ensayos de estos ensayos, no solamente aportan valores resistentes del material diseñado, si no que suponen adicionalmente una herramienta del control de ejecución de la propia estructura. Esta campaña se realiza tras la ejecución de la estructura del edificio, con la finalidad de que la ejecución de los prototipos reproduzca fielmente la situación real de la estructura, y con un doble objetivo. Por un lado, servirán para verificar el valor de la resistencia a tracción residual empleada en los cálculos, y por otro suponen una herramienta de control de ejecución acotado de la estructura ejecutada, ya que cada loseta circular se encuentra asociada a una de las plantas del edificio.

\section{DESCRIPCIÓN DE LA ESTRUCTURA DE ANÁLISIS}

La estructura portante se compone de pilares de hormigón armado, sobre los que apoyan directamente sin vigas, losas macizas de $300 \mathrm{~mm}$ de espesor correspondientes a los forjados de las cinco plantas del edificio. Las dimensiones del forjado en planta son de $33 \times 20 \mathrm{~m}$. La planta tipo de la construcción, se desarrolla en torno a un núcleo central que contiene las comunicaciones del edificio, las instalaciones y los servicios, lo que supone la existencia de huecos en los forjados estructurales. En la Figura 1 se representa la planta estructural tipo del edificio. Los pilares se han dispuesto según cuatro alineaciones longitudinales $A, B, C$ y $D$, separadas $8 \mathrm{~m}, 4,5 \mathrm{~m}$ y 5,4 $\mathrm{m}$ respectivamente, y según cinco alineaciones transversales 1, 2, 3, 4 y 5, espaciadas 7,8 m. Los paños estructurales de mayor dimensión resultan ser retículas de $8 \times 7,8 \mathrm{~m}$, situadas entre las alineaciones $A$ y $B$. 


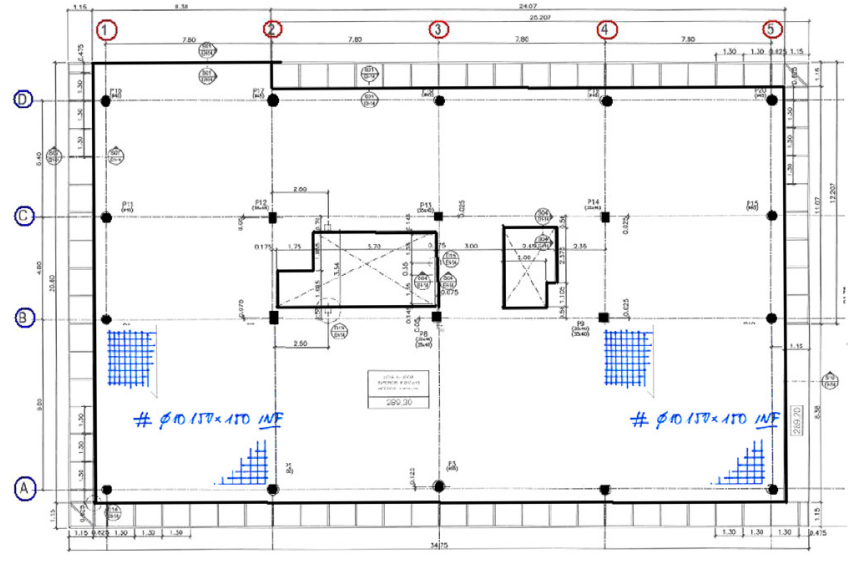

Figura 1. Planta estructural tipo.

El HRFA empleado se compone de una matriz de hormigón de resistencia característica a compresión simple de $30 \mathrm{~N} / \mathrm{mm}^{2}$, aunque con el fin de garantizar buena trabajabilidad y retracción mínima, así como evitar problemas durante su bombeo en obra, su dosificación cumple ciertas prescripciones. La fibras empleadas se corresponden con las del tipo ARCELOR TABIX 1.3/50, con sección circular de $1,3 \mathrm{~mm}$ de diámetro, $50 \mathrm{~mm}$ de longitud, y geometría ondulada en toda su longitud. La dosificación prevista expresada en peso de fibra metálica por unidad de volumen de hormigón es de $100 \mathrm{~kg} / \mathrm{m}^{3}$, y su adición se realiza en la planta de elaboración del hormigón.

En las retículas estructurales más solicitadas, se ha dispuesto de un emparrillado inferior de armadura pasiva convencional adicional a las fibras. De esta manera, el momento último resistente $M_{u}$ en régimen plástico, vendrá definido por la resistencia a tracción residual del HRFA y por el esfuerzo de agotamiento plástico de dicha armadura, permitiendo así alcanzar luces mayores en paños comprometidos como son los de esquina sin continuidad. Este refuerzo consiste en un emparrillado inferior de diámetro $10 \mathrm{~mm}$ cada $15 \mathrm{~cm}$ (Emp. Ø $10 \mathrm{c} / 15)$, de un acero corrugado de $500 \mathrm{~N} / \mathrm{mm}^{2}$ de límite elástico característico. En la Figura 2 se muestra el hormigonado de una de las plantas del edificio.

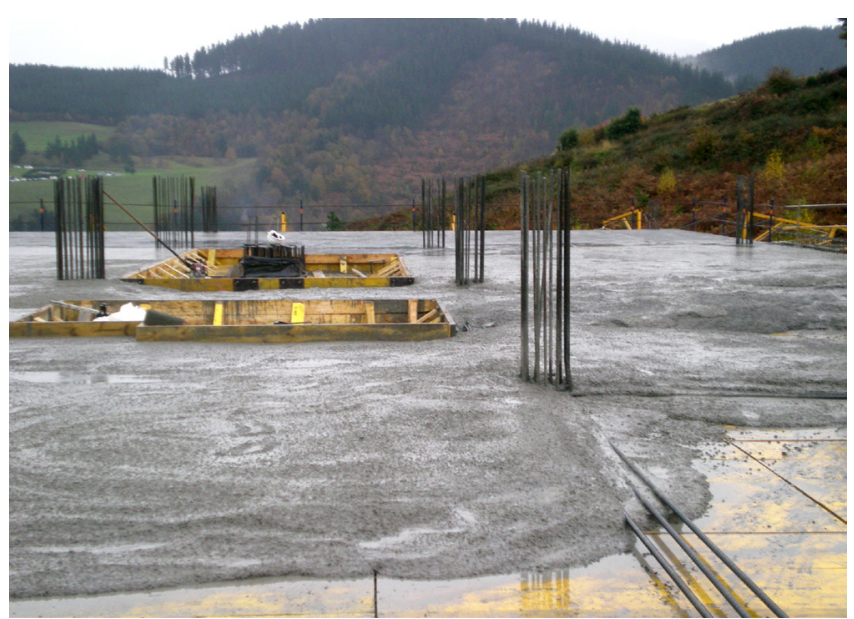

Figura 2. Hormigonado de planta estructural.

\section{CÁlCULO PLÁSTICO DE SECCIÓN DE HRFA}

En la obtención del momento último de la sección, se ha empleado el diagrama $\sigma-\varepsilon$ rectangular propuesto por EHE para el bloque de tracciones, mostrado en la Figura 3.

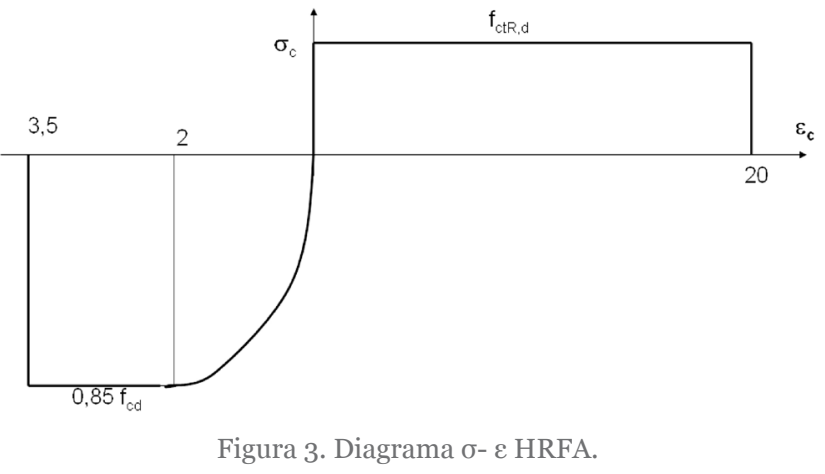

Para secciones solicitadas a flexión, como es el caso de las losas de forjado frente a cargas descendentes, la deformación máxima en tracción es del 20 por 1000, mientras que en compresión es del 3,5 por 1000. El valor máximo de la tensión en tracción es la resistencia residual a tracción de cálculo $f_{c t R, d}$. En compresión la tensión máxima es $0,85 f_{c d}$, siendo $f_{c d}$ la resistencia a compresión simple de cálculo del hormigón. Con el fin de simplificar los cálculos, en el bloque de compresiones se adopta finalmente el diagrama rectangular propuesto alternativamente por EHE.

El valor de la resistencia residual a tracción característica $f_{c t R}$ se ha fijado en $2,2 \mathrm{~N} / \mathrm{mm}^{2}$, y se asocia al valor máximo de la tensión a tracción, según el diagrama rectangular de la Figura 3. Este valor se ha obtenido de los trabajos basados en ensayos de rotura en prototipos realizados por (15) y (16), con dosificaciones y fibras similares a las empleadas en la estructura del presente trabajo. En la campaña experimental se verificará el valor adoptado.

Mediante la resistencia residual a tracción se evalúa el momento resistente plástico de la sección en estado límite último. Considerando un valor del coeficiente de minoración de resistencia $\gamma_{c}$ de 1,5, similar al empleado para el hormigón convencional, el valor de la resistencia residual a tracción de cálculo $f_{c t R, d}$ se define según [1]:

$$
f_{c t R, d}=\frac{f_{c t R}}{g_{c}}=\frac{2,2}{1,5}=1,46 \mathrm{~N} / \mathrm{mm}^{2}
$$

La distribución de tracciones y compresiones, en una sección de HRFA en estado límite último, y en general con armadura pasiva convencional, se muestra en la Figura 4. Se observa que en el bloque de tracciones, en la posición de la armadura pasiva, la deformación se limita al 20 por 1000, que es la deformación límite recomendada por EHE para el HRFA. El acero puede desarrollar deformaciones muy superiores a ésta hasta rotura, aunque se supone que la armadura alcanza su deformación máxima ya que se da por supuesto que ha plastificado, mientras que la deformación máxima $\varepsilon_{c}$ en la zona de compresiones, estará entre 2 y 3,5 por 1000.

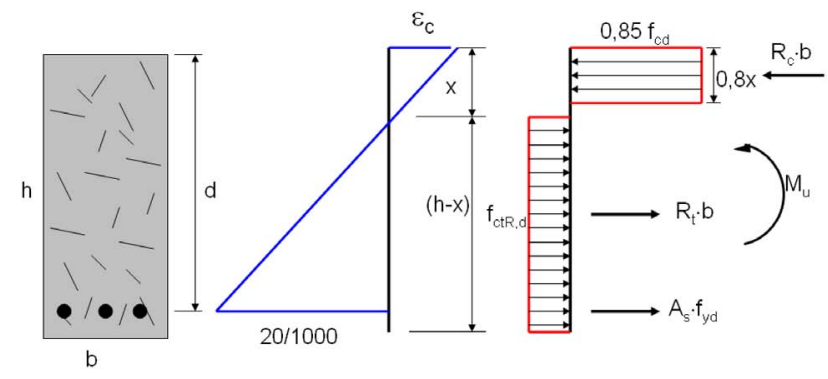

Figura 4. Distribución de esfuerzos en sección a flexión. 
En el caso de que la sección se encuentre solicitada a flexión pura, se cumple el equilibrio de fuerzas horizontales, por lo que igualando la resultante de compresiones a la de tracciones, se obtiene la profundidad «X» del eje neutro según [2]:

$$
x=\frac{f_{c t R, d} \cdot h \cdot b+A_{S} \cdot f_{y d}}{0,85 \cdot f_{c d} \cdot 0,8 \cdot b+f_{c t R, d} \cdot b}
$$

Las distancias de las resultantes, de los diferentes esfuerzos de sección, se representan en la Figura 5:

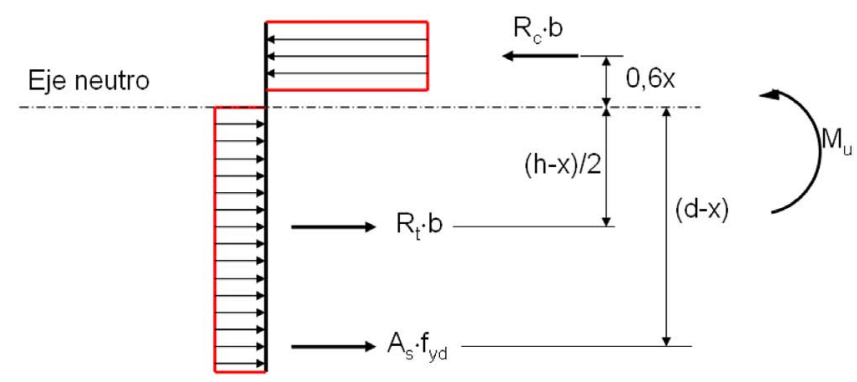

Figura 5. Situación de esfuerzos resultantes en flexión respecto a eje neutro.

Conocida la posición del eje neutro, y tomando momentos respecto a él, se obtiene el momento último de la sección según la expresión [3]:

$M_{u}=R_{c} \cdot b \cdot 0,6 \cdot x+R_{t} \cdot b \cdot \frac{(h-x)}{2}+A_{S} \cdot f_{y d} \cdot(d-x)$

Teniendo en cuenta los valores de cálculo de las características mecánicas de los materiales definidos, así como las expresiones [2] y [3], se obtienen los valores del momento último y las posiciones $x$ del eje neutro para las diferentes secciones de losa de la estructura. Los valores resultantes se incluyen en la Tabla 1.

Tabla 1. Secciones tipo. Valores resultantes en estado límite último.

\begin{tabular}{|l|l|c|}
\hline & $\begin{array}{c}\text { SECCIÓN 1 } \\
\text { HRFA }\end{array}$ & $\begin{array}{c}\text { SECCIÓN 2 } \\
\text { HRFA+ Emp. Ø 10 c/15 }\end{array}$ \\
\hline $\boldsymbol{x}$ & $29 \mathrm{~mm}$ & $44,28 \mathrm{~mm}$ \\
\hline $\boldsymbol{\varepsilon}_{\mathbf{c}}\left(\%_{\mathrm{o}}\right)$ & 2,14 & 3,46 \\
\hline$M_{\boldsymbol{u}}$ & $\mathbf{6 0 , 4} \mathbf{~ k N m} / \mathbf{m}$ & $\mathbf{1 1 6 , 5} \mathbf{~ k N m} / \mathbf{m}$ \\
\hline
\end{tabular}

El valor de $\varepsilon_{\mathrm{c}}$, como deformación máxima del bloque de compresiones, está comprendida en los dos casos entre 2 y 3,5 por 1000. Para placas macizas, se considera que las líneas de rotura tienen suficiente capacidad de rotación, y por lo tanto capacidad para redistribuir esfuerzos, si la profundidad de la fibra neutra es menor que $o, 3 d$, siendo d el canto útil de la sección. Esta condición se cumple en las dos secciones de estudio de la Tabla 1, donde la profundidad del eje neutro $x$ es menor que el $30 \%$ del canto útil. El canto útil se fija en 270 $\mathrm{mm}$, considerando un recubrimiento mecánico medio de la armadura pasiva convencional de $30 \mathrm{~mm}$.

Se concluye que las secciones de HRFA de la estructura descrita, tienen la capacidad de alcanzar un momento plástico último gracias a la resistencia residual en tracción, y además con una capacidad suficiente de rotación para ser asimiladas como rotulas plásticas.

\section{CÁlCULO PLÁSTICO dE LOSAS MEDIANTE LÍNEAS DE ROTURA}

El método de las líneas de rotura (14), permite la obtención de una relación entre la carga de colapso de una placa con unas determinadas condiciones de sustentación, y el momento último plástico que es capaz de soportar la sección. Una misma placa con unas determinas condiciones de apoyo, puede presentar múltiples combinaciones de líneas de rotura o configuraciones de rotura. A cada una de las posibles configuraciones de rotura le corresponde un mecanismo de colapso diferente, con su carga de colapso. La configuración verdadera es aquella que proporciona la carga de colapso más pequeña. De los métodos existentes para la obtención de la carga de colapso a partir de una configuración de rotura, se ha optado por el método del equilibrio.

Existen referencias bibliográficas que presentan formularios para el cálculo de placas con apoyos lineales, mediante el método plástico de las líneas de rotura, que incluyen tablas prácticas para el proyecto de placas rectangulares (17). En lo que respecta a placas continuas con apoyos puntuales, como la estructura descrita en este trabajo, no se dispone de formularios que permitan abordarlas por el método de las líneas de rotura. Ello es debido fundamentalmente a que los ejes de rotación de los trozos de placas no están prefijados de antemano, lo que supone el análisis de un mayor número de configuraciones de rotura y de posibles mecanismos de colapso.

Los posibles mecanismos de colapso de la estructura objeto de estudio se han agrupado en tres casos de configuraciones de rotura para cargas superficiales. En las Figuras 6, 7 y 8 se representan respectivamente las configuraciones de líneas de rotura A, B y C. Se observan líneas de rotura positivas y negativas, y los momentos constantes actuantes en cada una de ellas. Los momentos de líneas de rotura positivas correspondientes a las retículas de esquina $A B 12$ y $A B 45$ se han denotado como $m$ ', y se corresponden con el momento plástico último de la sección de HRFA mas un emparrillado inferior de diámetro $10 \mathrm{~mm}$ cada $15 \mathrm{~cm}$. En el resto de las líneas de rotura, el momento $m$ se corresponde con el momento último plástico de la sección de HRFA sin armadura convencional adicional. Los valores de ambos momentos son conocidos y se han obtenido en la tabla 1 del apartado anterior.

En la configuración de líneas de rotura A, se presentan básicamente cuatro tipos de mecanismos de rotura parciales. El mecanismo de rotura parcial que divide la losa en trozos de tipo 1/2/3 se da en las retículas de esquina $A B 12$ y $A B 45$, en cuyo caso actúa el momento $m$ ' en las líneas de rotura positivas, mientras que en las negativas actúa $m$. En las otras dos retículas en esquina de la planta se da la rotura parcial de los trozos tipo 4/5/6, con una forma similar a la anterior aunque con luces distintas, y actuando en este caso el momento plástico último $\mathrm{m}$ tanto en las líneas de rotura positivas como en las negativas. Un tercer mecanismo de rotura es el que divide las retículas centrales de 7,8 m de luz en trozos tipo $7 / 8$, y por último se encuentra la rotura parcial asociada a las retículas centrales de 4,5 $\mathrm{m}$ de luz que genera los trozos tipo 9/10. 


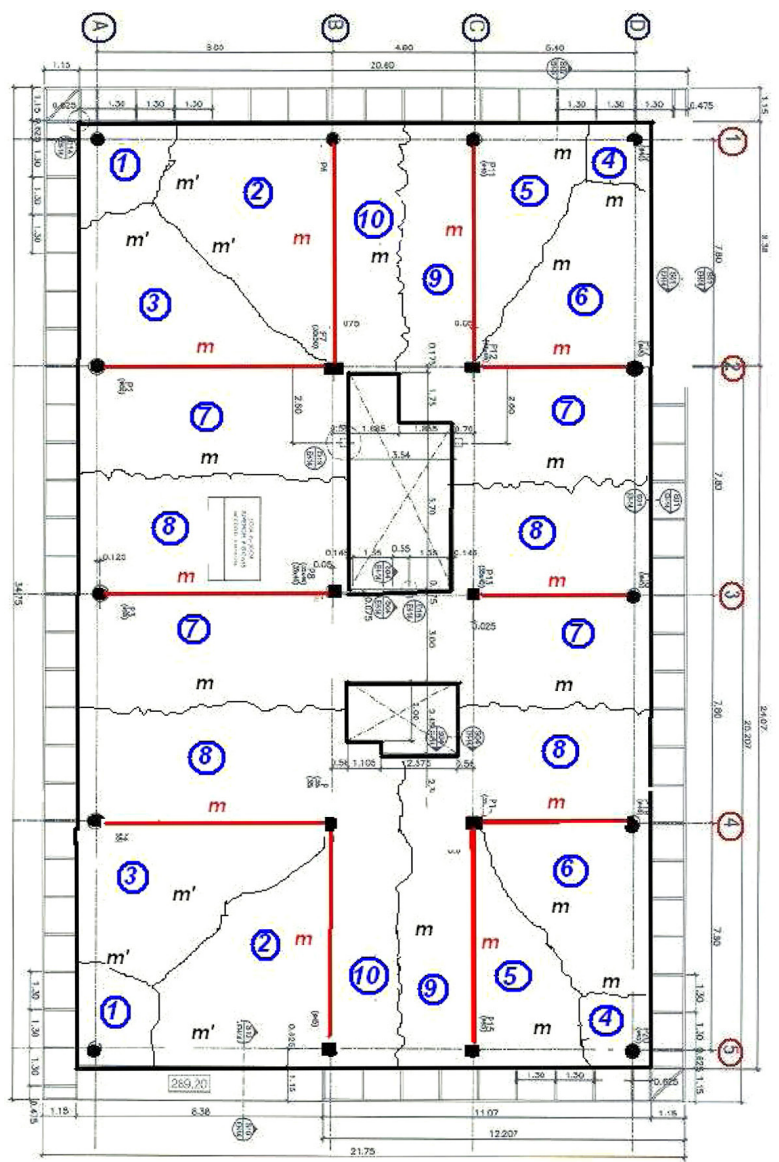

Figura 6. Configuración de líneas de rotura A.

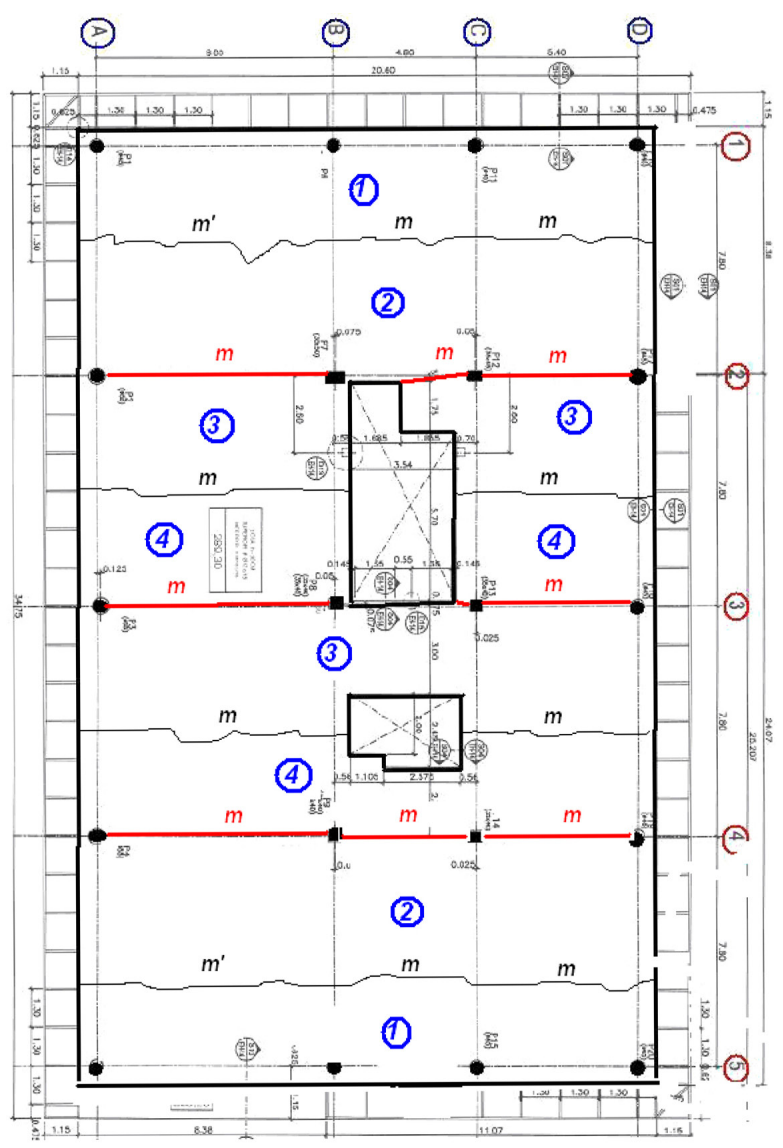

Figura 7. Configuración de líneas de rotura B.

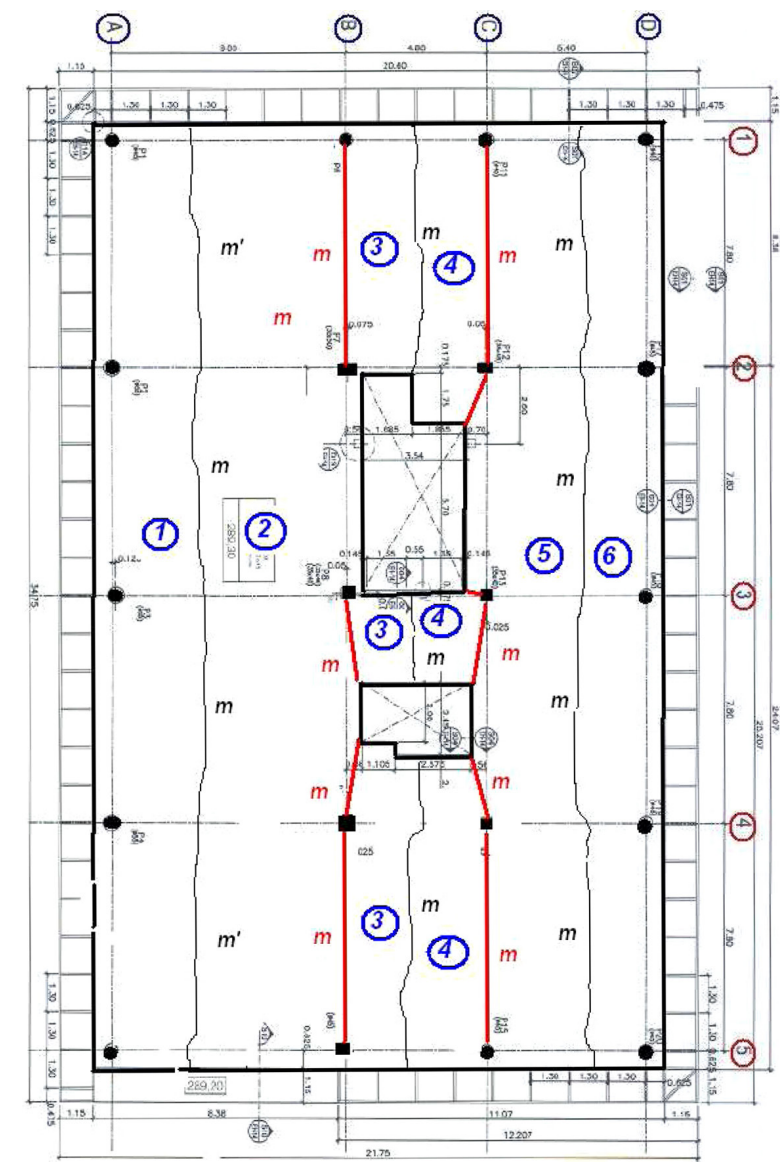

Figura 8. Configuración de líneas de rotura C.

En la configuración de líneas de rotura B mostrada en la Figura 7 se han previsto únicamente líneas de rotura horizontales. En esta segunda configuración se distinguen básicamente dos tipos de mecanismos de rotura parcial, la rotura parcial de los trozos 1/2, y la de los trozos tipo $3 / 4$.

En la configuración de líneas de rotura C, mostrada en la Figura 8 , se han previsto líneas de rotura verticales. Se distinguen tres mecanismos de rotura parcial. El primero de ellos, que separa la losa en trozos tipo 1/2, se produce entre las alineaciones transversales $A, B$, y en él actúa el momento $m$ ' en las líneas de rotura positivas de las retículas de esquina, debido al refuerzo de armadura inferior convencional. Otro mecanismo de rotura parcial divide a la losa en trozos tipo 3/4, coincidiendo este caso con uno de los mecanismos parciales de la configuración denominada como A. Por último entre las alineaciones transversales $C, D$ se da un tercer mecanismo asociado a los trozos de losa 5/6.

Se han resuelto las diferentes configuraciones de rotura parciales por el método de equilibrio obteniéndose en cada caso la relación $m / q$. En la tabla 2 se muestran los resultados obtenidos para las diferentes configuraciones y mecanismos de rotura parcial. En la tercera columna se incluyen los valores de las cargas últimas de colapso $q_{\mathrm{u}}$ obtenidas.

En la Tabla 2 se observa que la máxima relación $m / q$ la proporciona el mecanismo de rotura parcial de los trozos tipo $1 / 2$ de la configuración C. La carga minima de colapso $q_{u}, \mathrm{y}$ por lo tanto la real, es de $14,15 \mathrm{kN} / \mathrm{m}^{2}$. El valor de la carga actuante de cálculo $q_{d}$ se corresponde con un peso propio de 
Tabla 2. Relación $m / q$ y cargas ultimas de colapso para cada uno de los mecanismos de rotura parcial.

\begin{tabular}{|l|c|c|}
\hline \multicolumn{3}{|c|}{ CONFIGURACIÓN DE LÍNEAS DE ROTURA A } \\
\hline Mecanismo de rotura parcial & $\mathbf{m} / \mathbf{q}$ & $\begin{array}{r}\mathbf{q}_{\mathbf{u}} \\
\left(\mathbf{k N} / \mathbf{m}^{\mathbf{2}}\right)\end{array}$ \\
\hline Rotura parcial 1/2/3 & 2,42 & 24,95 \\
\hline Rotura parcial 4/5/6 & 2,37 & 25,47 \\
\hline Rotura parcial 7/8 & 3,8 & 15,88 \\
\hline Rotura parcial 9/10 & 1,27 & 47,56 \\
\hline \multicolumn{2}{|c|}{ CONFIGURACIÓN DE LÍNEAS DE ROTURA B } \\
\hline Mecanismo de rotura parcial & m/q & $\begin{array}{r}\mathbf{q}_{\mathbf{u}} \\
\left(\mathbf{k N} / \mathbf{m}^{2}\right)\end{array}$ \\
\hline Rotura parcial 1/2 & 4,09 & 14,76 \\
\hline Rotura parcial 3/4 & 3,8 & 15,88 \\
\hline CONFIGURACIÓN DE LíNEAS DE ROTURA C \\
\hline Mecanismo de rotura parcial & m/q & $\mathbf{q}_{\mathbf{u}}$ \\
\hline Rotura parcial 1/2 & 4,26 & 14,15 \\
\hline Rotura parcial 3/4 & 1,27 & 47,56 \\
\hline Rotura parcial 5/6 & 2,69 & 22,44 \\
\hline
\end{tabular}

$7,5 \mathrm{kN} / \mathrm{m}^{2}$, una tabiquería equivalente a una carga superficial de $1 \mathrm{kN} / \mathrm{m}^{2}$ y un solado de $0,2 \mathrm{kN} / \mathrm{m}^{2}$. La sobrecarga de uso es de $2 \mathrm{kN} / \mathrm{m}^{2}$, aunque se encuentra atenuada por un coeficiente reductor de sobrecargas de 0,7, debido a que el mecanismo de fallo indicado se refiere a un elemento portante superficial que supera $100 \mathrm{~m}^{2}$. La carga de cálculo, con los correspondientes coeficientes de mayoración, se obtiene según [4]:

$$
q_{d}=1,35 \cdot(7,5+1+0,2)+1,5 \cdot 0,7 \cdot(2)=13,845 \mathrm{kN} / \mathrm{m}^{2}
$$

Se concluye que el valor de la carga actuante $q_{d}$ no supera el valor de la carga resistente última $q_{u}$ de $14,15 \mathrm{kN} / \mathrm{m}^{2}$.

\section{ENSAYO DE PROTOTIPOS}

La campaña experimental posee un doble objetivo. Por un lado pretende verificar la resistencia de referencia empleada en los cálculos, que ha sido tomada de las experiencias de los autores referenciados anteriormente. Por otro lado es un control de calidad de la estructura ejecutada, ya que los prototipos se han elaborado in situ durante la propia ejecución de las losas de forjado del edificio piloto, como se observa en la Figura 9. Los materiales empleados, medios de ejecución y condiciones de curado son idénticos. Por lo tanto, los resultados de los ensayos constituyen un control de calidad de ejecución de la estructura, ya que cada uno de los prototipos se encuentra asociado a una planta del edificio, coincidiendo su elaboración con el día de hormigonado de cada planta.

A partir de los ensayos, mediante una correlación de líneas de rotura, es posible obtener el momento plástico último $M_{u}$ de la sección de las losetas asociado a la carga máxima y a la formación de un mecanismo de colapso plástico. A partir del $M_{u}$, considerando una distribución plástica de tensiones en la sección de HRFA, se obtiene el valor de la resistencia residual a tracción $f_{c t R}$.

Los prototipos a ensayar en laboratorio consisten en seis losas circulares de $2.200 \mathrm{~mm}$ de diámetro y $200 \mathrm{~mm}$ de espesor, fabricadas en HRFA de idénticas características que

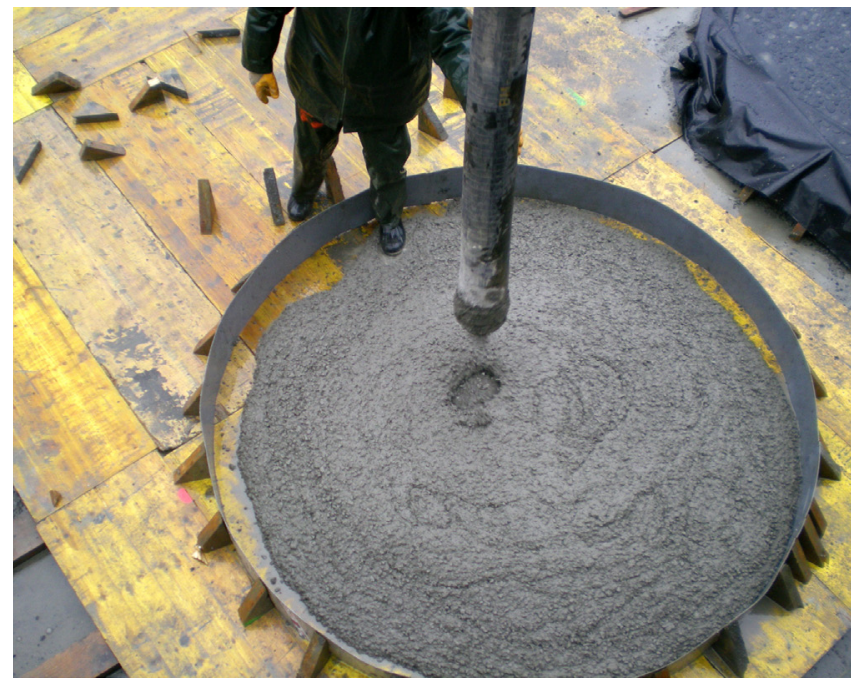

Figura 9. Hormigonado de prototipos loseta circular.

las losas de la estructura descrita anteriormente. El apoyo de cada loseta durante el ensayo es continuo y circunferencial a lo largo de todo su perímetro, tal y como se muestra en la Figura 10. La luz libre diametral de cada prototipo debe ser de $2.000 \mathrm{~mm}$, por lo que la anchura del apoyo perimetral es de $100 \mathrm{~mm}$. Las losetas se someten a una carga central concentrada mediante una prensa, trabajando con descenso controlado mediante un sistema retroalimentado en bucle cerrado. La carga actuante se transmite al centro geométrico de la loseta a través de un útil cilíndrico sólido de acero de 200 $\mathrm{mm}$ de diámetro. El registro de datos se prolonga hasta una deformación total vertical de la loseta de $40 \mathrm{~mm}$.

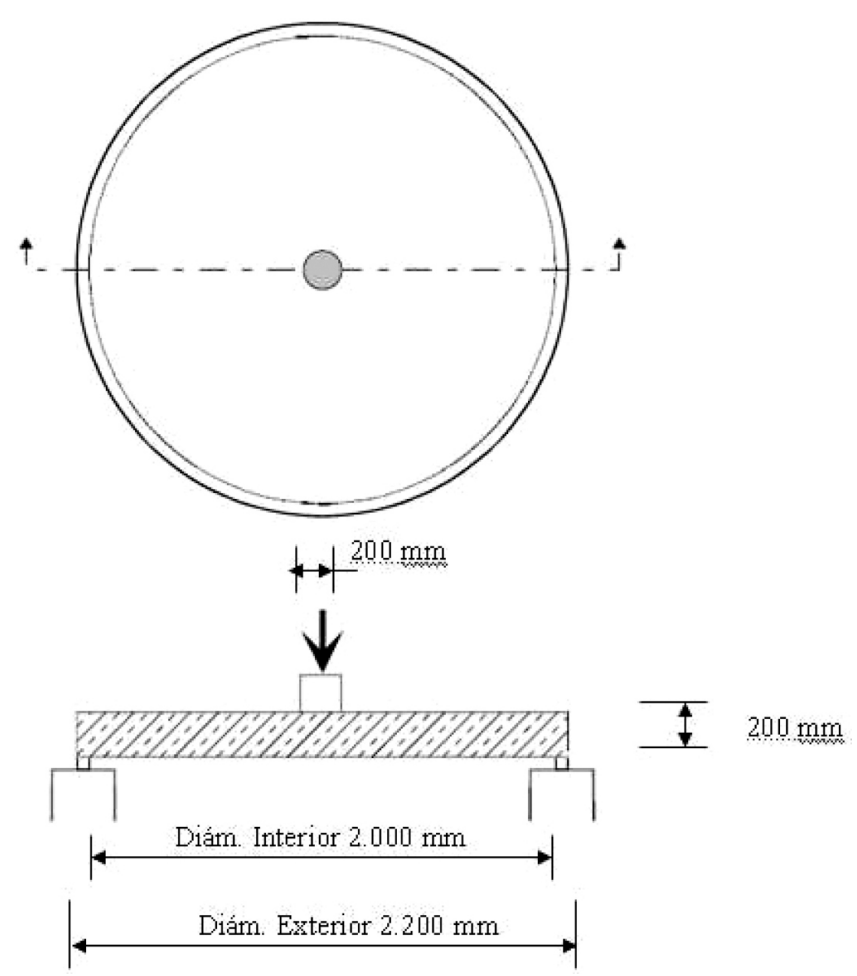

Figura 10. Esquema de ensayo de losetas circulares.

En la Figura 11 se observa la forma de rotura de los prototipos ensayados. Se observan líneas de rotura principales y secundarias. 


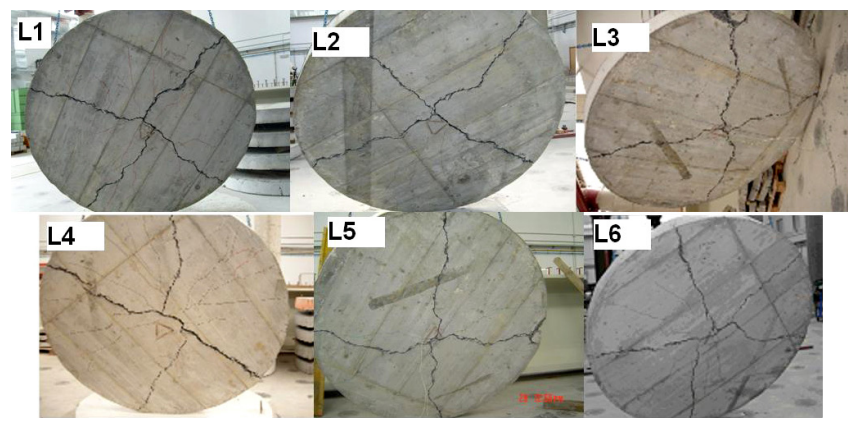

Figura 11. Líneas de rotura en prototipos ensayados.

Para cada una de las losetas ensayadas se ha obtenido la gráfica de deformación vertical del útil cilíndrico en el eje de abcisas ( $\mathrm{mm}$ ) y carga aplicada por el actuador en el eje de ordenadas $(\mathrm{kN})$. Las gráficas, así como la carga máxima alcanzada en cada prototipo se muestran en la Figura 12.

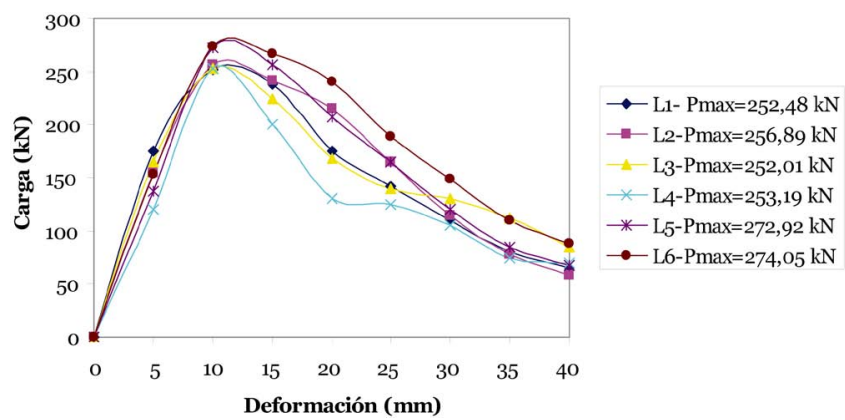

Figura 12. Gráfica carga- deformación de losetas circulares.

La parte inicial de la curva de ensayo muestra un comportamiento lineal hasta el inicio de la fisuración. Tras el inicio de la fisuración y plastificación de secciones, comienza el desarrollo de líneas de rotura hasta la formación de un mecanismo de colapso asociado a la carga máxima. Existe una relación entre el número de líneas de rotura radiales que se producen y la carga máxima $P_{\max }$ que se alcanza en el ensayo. La loseta L6, que presenta cinco líneas de rotura principales y múltiples secundarias, proporciona el valor máximo de la carga. El resto de prototipos presentan cuatro líneas de rotura radiales principales. A lo largo de las líneas de rotura actúa un momento constante que es el momento de plastificación de la sección. La rotura de los prototipos se produce de manera progresiva y sin fragilidad. Al final del ensayo, y correspondiente a un desplazamiento vertical de $40 \mathrm{~mm}$, existe una carga remanente. El valor de esta carga remanente es de un $30 \%$ del valor de la carga máxima $P_{\max }$.
La relación mediante el método de las líneas de rotura, entre la carga máxima concentrada $P_{\max }$ y el momento último plástico $M_{u}$ de la sección se indica en [5]. El peso propio de la loseta se corresponde con $q_{p p}, R$ es el radio de la loseta y $a$ es el radio del útil de aplicación de la carga.

$$
M_{u}=\frac{q_{p p} R^{2}}{6}+\frac{P_{\max }}{2 \pi}\left(1-\frac{2 a}{3 R}\right)
$$

A nivel de sección, actúa un momento plástico con distribución de tensiones rectangular, tanto en el bloque de tracciones como de compresiones, donde la profundidad del eje neutro es el 10 \% del canto de la sección. En esta situación, el valor de la resistencia residual a tracción $f_{c t R}$ viene dado por [6] según Soranakom et ál (6).

$$
f_{c t R}=\frac{M_{u}}{0,45 h^{2}}
$$

La Tabla 3 incluye los valores de resistencia a tracción residual obtenidos a partir del valor de la carga máxima $P_{\max }$ de los ensayos de losetas circulares. El valor del momento plástico último $M_{u}$ se ha obtenido mediante [5], y la resistencia a tracción residual experimental ${ }_{\text {fctR,exp }}$ mediante [6]. Los valores de referencia para esta resistencia, adoptados en el cálculo plástico de la sección son de $2,2 \mathrm{~N} / \mathrm{mm}^{2}$ como valor característico $f_{\text {ctR }}$ y de $1,46 \mathrm{~N} / \mathrm{mm}^{2}$ como valor de cálculo $f_{c t R, d}$. En las dos últimas columnas de la tabla se indican, en porcentaje, la relación entre el valor experimental obtenido y el valor de referencia característico y de cálculo empleados originalmente.

El valor medio de la resistencia a tracción residual experimental es de 2,196 N/mm². Supone un $99 \%$ del valor característico de referencia de $2,2 \mathrm{~N} / \mathrm{mm}^{2}$ tomado en los cálculos. Respecto al valor de cálculo de $1,46 \mathrm{~N} / \mathrm{mm}^{2}$ empleado en las comprobaciones estructurales, el valor medio de los resultados experimentales lo supera en un 50 \%. El valor mínimo obtenido, correspondiente a la loseta L3, supera al valor de cálculo en un $46 \%$. La dispersión máxima de resultados es inferior al $5 \%$.

\section{CONCLUSIONES}

El análisis plástico aplicado a losas de forjado de HRFA apoyadas directamente sobre pilares, permite un mejor aprovechamiento del material. Sin embargo requiere el análisis mediante el método de las líneas de rotura de las múltiples configuraciones de colapso, incluyendo mecanismos de rotu-

Tabla 3. Resistencia a tracción residual experimental en ensayos de prototipos.

\begin{tabular}{|l|c|c|c|c|c|}
\hline \multicolumn{7}{|c|}{ RESISTENCIA A TRACCIÓN RESIDUAL $\left(\boldsymbol{f}_{\text {ctR }}\right)$} \\
\hline $\begin{array}{c}\mathbf{N}^{\mathbf{0}} \mathbf{d e} \\
\text { Prototipo }\end{array}$ & $\begin{array}{c}\boldsymbol{P}_{\text {max }} \\
(\mathbf{k N})\end{array}$ & $\begin{array}{c}\boldsymbol{M}_{\boldsymbol{u}} \\
(\mathbf{k N m} / \mathbf{m})\end{array}$ & $\begin{array}{c}\boldsymbol{f}_{\text {ctR,exp }} \\
\left(\mathbf{N} / \mathbf{m m}^{\mathbf{2}}\right)\end{array}$ & $\frac{\boldsymbol{f}_{\text {ctR, } \mathbf{e x p}}}{\boldsymbol{f}_{\text {ctR }}} \%$ & $\frac{\boldsymbol{f}_{\text {ctR, exp }}}{\boldsymbol{f}_{\text {ctR, }}} \%$ \\
\hline L1 & 252,48 & 38,34 & 2,13 & 97 & 146 \\
\hline L2 & 256,89 & 38,99 & 2,17 & 98 & 148 \\
\hline L3 & 252,01 & 38,26 & 2,13 & 97 & 146 \\
\hline L4 & 253,19 & 38,44 & 2,14 & 97 & 146 \\
\hline L5 & 272,92 & 41,37 & 2,30 & 104 & 157 \\
\hline L6 & 274,05 & 41,55 & 2,31 & 104 & 158 \\
\hline V. Medio & $\mathbf{2 6 0 , 2 5}$ & $\mathbf{3 9 , 4 9}$ & $\mathbf{2 , 1 9 6}$ & $\mathbf{9 9} \%$ & $\mathbf{1 5 0} \%$ \\
\hline
\end{tabular}


ra parcial, para obtener la carga real de colapso de la estructura. Este estudio se ha realizado sobre la estructura de un edificio real construido en HRFA, y se ha comprobado que el valor de la carga de colapso superficial de cada retícula tipo es mayor que la carga de diseño.

En el cálculo plástico de sección de HRFA, se emplea un diagrama plástico- perfecto para el bloque de tracciones, aunque ello requiere evaluar o conocer previamente la resistencia residual a tracción del material. La verificación de esta resistencia residual se ha realizado mediante el ensayo de seis prototipos en forma de loseta circular de $2 \mathrm{~m}$ de diámetro y $200 \mathrm{~mm}$ de espesor, construidos con el mismo material y en las mismas condiciones que la estructura objeto de estudio.

La rotura de los prototipos se produce de manera progresiva y sin fragilidad. Al final del ensayo, existe una carga remanente del $30 \%$ del valor de la carga máxima. Mediante una correlación basada en el método de análisis plástico de líneas de rotura, se relaciona la carga máxima del ensayo con la resistencia residual a tracción experimental. El valor medio de la resistencia a tracción residual experimental, supone un $99 \%$ del valor característico de referencia tomado en los cálculos. Respecto al valor de cálculo empleado en las comprobaciones estructurales, el valor medio de los resultados experimentales representa un $150 \%$. La dispersión de resultados es inferior al $5 \%$, que es sensiblemente inferior a la que presentan tradicionalmente los ensayos de probetas prismáticas de HRFA.

Los prototipos se han elaborado y curado en obra, a la intemperie, y en definitiva con los mismos materiales, medios y condiciones que la estructura construida. Desde el punto de vista de control de ejecución de las losas de HRFA del edificio, y teniendo en cuenta que todas las resistencias obtenidas superan al valor de referencia de cálculo, se consideran resultados satisfactorios.

\section{AGRADECIMIENTOS}

Los autores agradecen el apoyo aportado por LKS en el desarrollo de este trabajo, como promotor e impulsor de esta experiencia. Asimismo agradecen al Gobierno Vasco su ayuda IT-453-10 y a la UPV/EHU por su ayuda Unidad de Formación e Investigación UFI11/29.

\section{REFERENCIAS}

(1) Blanco, A. (2008). Durabilidad del hormigón con fibras de acero (Tesina de especialidad. Dir. A. Aguado de Cea y M. Vandewalle). Barcelona: UPC.

(2) Barros, J. A. O., Figuieras, J. A. (1999). Flexural behaviour of SFRC: testing and modelling. Journal of Materials in Civil Engineering, 11(4): 331-339, doi: http://dx.doi.org/10.1061/(ASCE)0899-1561(1999)11:4(331).

(3) Lambrechts, A. (2007, 23 de mayo). Self compacting steel fibre concrete, and its use in segmental lining. En Avances en tecnología del hormigón en construcción subterránea, Jornada Técnica 2007-JT-01, (pp. 53-68). Barcelona: Departamento de Ingeniería de la Construcción, ETS Ingenieros de Caminos, Canales y Puertos - UPC.

(4) Massicotte, B., Moffat, K. (2003). Developpments pour l'utilisation des fibres dans des applications structurale. Canadá: Departament des genies civil, geoloique et mines -Universidad de Montreal.

(5) Gossla, U. (2005). Development of SFRC Free Suspended Elevated Flat Slabs. Alemania: Dept. of Civil Engineering Aachen University of Applied Sciences.

(6) Soranakom, C., Mobasher, B., Destrée, X. (2007, 27 de marzo). Elevated Slabs with Steel Fibre Reinforced Concrete: Full scale testing, and Inverse Analysis of FRC Round Panel Tests. ACI Materials Journal.

(7) Gossla, U. (2007). Development of SFRC Flat Slabs. Part II: Column Settlement and Punching Shear Test. Alemania: Dept. of Civil Engineering, Aachen University of Applied Sciences.

(8) Alavedra, P., Destrée, X., González, J.R., Osa, A. (2005). Utilización de hormigón armado con alta dosificación de fibres de acero en la rehabilitación de un edificio industrial . En III Congreso de ACHE de puentes y estructuras.

(9) Aguado, A., Marí, A., Molins, C. (2005). Estudio de viabilidad del ensayo Barcelona. En III Congreso de Ache de puentes y estructuras. Zaragoza.

(10) Saludes, S., Aguado, A., Molins, C. (2006). Ensayo de doble punzonamiento aplicado al hormigón reforzado con fibras (ensayo Barcelona). Barcelona: Publicacions del department d'enginyeria de la contrucció, ETSECCPB-UPC.

(11) Bernard, E. S. (2002). Correlations in the behavior of fibre reinforced shotcrete beam and panel specimen. Materials and Structures, 35(3): 156-164, doi: http://dx.doi.org/10.1007/BFo2533584.

(12) Blanco, A., Pujadas, P., de la Fuente, A., Aguado, A. (2010). Análisis comparativo de los modelos constitutivos del hormigón reforzado con fibras. Hormigón y Acero, 61(256): 83-101.

(13) Ministerio de fomento. (2008). Instrucción del Hormigón Estructural, EHE o8. Madrid: Ministerio de fomento.

(14) Johansen, K. W. (1972). Yield line Formulae for Slabs. London: Cement and Concrete Association.

(15) Espion, B., Destrée, X. (2004). Test report of loading up to failure of five fiber reinforced concrete (FRC) slabs. Faculte de Genie Civil. Universite Libre de Bruxelles. Brussels.

(16) Germain, O. Pierre, C. (2007). Punching tests of three fibre reinforced concrete slabs coming from Talien (Estonia). Brussels : Centre de Recherche de L'Industrie Cimentaire (CRIC).

(17) CEB. Code Modele CEB-FIP pour structures en beton. (1978). Boletin de Informacion del CEB, $\mathrm{n}^{\circ}$ 124/125-F. Paris. 